\section{LA SEXUALIDAD EN ENTREDICHO: NUEVAS NEGOCIACIONES DEL SIGNIFICADO DE SER MUJER EN EL DEPORTE DE ALTO RENDIMIENTO}

\author{
SEXUALIDADE SOB SUSPEITA: NOVAS NEGOCIAÇÕES \\ DO SIGNIFICADO DE SER MULHER NO ESPORTE DE ALTO \\ DESEMPENHO
}

\section{SEXUALITY IN QUESTION: NEW NEGOTIATIONS OF THE MEANING OF BEING A WOMAN IN HIGH PERFORMANCE SPORTS}

\author{
Belén Donoso Pérez*, Amalia Reina Giménez*, \\ Alberto Álvarez-Sotomayor Posadillo*
}

Palavras chave: Sexualidade. Pesquisa Qualitativa. Identidade de Gênero. Feminilidade.

Keywords: Sexuality. Qualitative Research. Gender Identity. Femininity.
Resumen: Esta investigación tiene como objetivos, por un lado, explorar las percepciones de las deportistas acerca de cómo son evaluadas por su entorno social con relación a la feminidad y a su orientación sexual por su vinculación con una práctica deportiva de alto rendimiento. Por otro, analizar la visibilidad y aceptación de la homosexualidad en el contexto deportivo tanto por las deportistas como por los agentes sociales que las rodean. La metodología fue de tipo cualitativo. Se llevaron a cabo cinco grupos focales en los que participaron 46 mujeres practicantes de futbol sala, balonmano, voleibol, atletismo y natación. Los resultados muestran que las deportistas presentan un discurso de resistencia frente al estereotipo de marimacho-lesbiana que, comprobamos, continúa vigente en el imaginario colectivo. A pesar de que se perciben avances sociales en este sentido, la homosexualidad sigue estigmatizada y aún persisten formas de rechazo cognitivo-afectivo en parte de la sociedad española.

Resumo: Esta pesquisa tem como objetivo, por um lado, explorar as percepções das atletas sobre como são avaliadas pelo seu ambiente social em relação à feminilidade e sua orientação sexual, para vincular-se a uma prática esportiva de alto desempenho e, por outro, analisar a visibilidade e aceitação da homossexualidade no contexto esportivo, tanto pelos atletas como pelos agentes sociais ao seu redor. A metodologia foi qualitativa. Foram feitos cinco grupos focais nos quais participaram 46 mulheres praticando futsal, handebol, vôlei, atletismo e natação. Os resultados mostram que as atletas apresentam um discurso de resistência contra o estereótipo da "Maria-João" o "sapatão" que, verificamos, ainda está em vigor na imaginação coletiva. Embora os avanços sociais sejam percebidos nesse sentido, a homossexualidade permanece estigmatizada e ainda persistem as formas de rejeição cognitivo-afetiva em parte da sociedade espanhola.

Abstract: On the one hand, this research aims to explore the perceptions of women practicing high-performance sports about how they are viewed by their social environment in terms of their femininity and sexual orientation. On the other hand, it analyzes the visibility and acceptance of homosexuality in the sports context by both athletes and the social agents surrounding them. The research methodology was qualitative, with five focus groups including 46 women who practiced futsal, handball, volleyball, athletics and swimming. Results show that they present a resistance-oriented discourse against butch-lesbian stereotypes that, as we prove, are still present in collective imagination. Despite social advances perceived in this regard, homosexuality remains stigmatized and forms of cognitive-affective rejection persist in part of Spanish society.
*Universidad de Córdoba, Córdoba, España.

E-mail:

belendonosoperez@gmail.com; amalia.reina@uco.es; aasotomayor@uco.es

Recebido em: 17-09-2019 Aprovado em: 22-02-2020 Publicado em: 30-03-2020 


\section{INTRODUCCIÓN}

Desde sus orígenes, el deporte ha sido construido sobre la base de unos valores y normas consideradas masculinas tales como la fuerza, la agresividad o el poder, que, en la mayoría de las ocasiones, entran en contradicción con los roles de género femeninos tradicionales (PALZKILL; FISHER, 1990; FASTING; SCRANTON, 1997). En consecuencia, las mujeres que se han atrevido a participar en deportes de competición han encontrado que con frecuencia su sexualidad ha sido puesta en entredicho y se han visto devaluadas, sexualizadas y discriminadas por la sociedad. Paralelamente, el contexto deportivo se ha considerado como un espacio en el que se fomenta la heteronormatividad y en el que se decide lo que es masculino y femenino (LENSKYI, 1994; DOWLING, 2013). Quizás por esta razón siempre ha existido una cierta hostilidad hacia la atleta que se atreve a cuestionar las normas de género (SYKES, 1998; KAUER; KRANE, 2006).

Es evidente que las actitudes hacia las personas LGTB fluctúan según los contextos culturales (MARTíN; SOLER; VILANOVA, 2017). Concretamente, en el caso de las mujeres que practican deportes considerados tradicionalmente masculinos, suele surgir el prejuicio de que son homosexuales, debido, entre otras razones, a que han sido socializadas en un contexto en el que persisten actitudes negativas hacia la homosexualidad sostenidas por creencias culturales (KRANE, 1997). Estas creencias se desarrollan a través de una compleja interrelación de experiencias de aprendizaje, estrategias psicológicas de defensa de cada individuo y factores históricos y socioculturales (REMAFEDI, 1987). Como resultado de todo ello, es muy difícil que los individuos no tengan un cierto nivel de homofobia interiorizado (JAEGER et al. 2019). Sin embargo, aunque en la actualidad es más común usar el término homofobia, que se identifica como un miedo irracional e intolerancia hacia las lesbianas y los hombres gay, preferimos asumir el término homonegativismo (KRANE, 1997), referido a los estereotipos negativos, prejuicios y discriminación en contra de las personas que no son heterosexuales. Los individuos que han sufrido experiencias homonegativas, pueden presentar problemas de autoestima, padecer experiencias de rechazo, tener dificultades en el proceso de construcción de una identidad sexual positiva (HALBROOK; WATSON; VOELKER, 2018) tolerar niveles de estrés emocional añadido más elevados (JUSTER, 2016) y presentar mayor número de episodios de ansiedad y depresión (ROBINSON; ESPELAGE; RIVERS, 2013) comparados con sus pares heterosexuales.

Algunos estudios apuntan a que las mujeres desarrollan con más frecuencia que los hombres relaciones con personas de su mismo sexo durante la adolescencia tardía o cuando son adultas jóvenes (GROV et al., 2006). En ese sentido, las chicas adolescentes pueden utilizar el deporte para explorar la formación de su identidad, buscar a chicas de diversa orientación sexual o para disfrutar de un espacio en el que transgredir los roles y expectativas de género (KIVEL; KLEIBER, 2000).

La relación entre feminidad, sexualidad y el deporte de alto rendimiento socialmente etiquetado como masculino cuando es practicado por mujeres, ha estado presente en muchos estudios recogidos en la literatura internacional (GRIFFIN, 
1992b; HARGREAVES, 1997; HOWE, 2001; HARRIS, 2005; RAVEL; RAIL, 2006; OXFORD; MCLACHLAN, 2018). A pesar de que el deporte se ha constituido como un terreno masculino y discriminatorio, son numerosas las investigadoras/es que han encontrado que las mujeres revelan su verdadera orientación sexual en el entorno deportivo en el que se desenvuelven, concretamente con más frecuencia en los deportes colectivos, porque consideran al equipo como un refugio que les aporta seguridad y tranquilidad para entender, poner en orden los sentimientos o explorar con los que no han podido dar respuesta en otros escenarios (PALZKILL; FISHER, 1990; CAHN, 1993; STOELTING, 2011; FINK et al. 2012; MELTON; CUNNINGHAM, 2012; RATTS, 2013; DAVIS-DELANO, 2014; FYNES; FISHER, 2016).

No obstante, esta cuestión apenas ha sido objeto de investigación en España (MARTÍN; SOLER; VILANOVA, 2017). A pesar de que existen indicios de que la situación social de estas deportistas puede estar cambiando, la falta de estudios que examinen esta problemática en la sociedad española no permite realizar una valoración rigurosa (PIEDRA, 2015). Consecuentemente, la actualidad que implica este contexto hace que sean necesarias investigaciones como la que se presenta a continuación, que contribuyan a llenar el vacío empírico existente.

Este trabajo tiene como objetivos, por un lado, indagar en las percepciones que tienen las deportistas acerca de cómo son evaluadas por su entorno social con relación a la feminidad hegemónica y a su orientación sexual, por su vínculo con una práctica deportiva de alto rendimiento. Por otro lado, se analizan sus percepciones en torno a la situación de visibilidad y aceptación de la homosexualidad en el contexto deportivo y sobre el papel que juegan sus familias como principal agente de socialización.

En este sentido, los relatos de las deportistas seleccionadas muestran los rasgos discursivos comunes en relación a los objetivos planteados, contribuyendo de forma específica al campo de estudios de sexualidad y deporte en España.

\section{METODOLOGÍA}

\subsection{PARTICIPANTES}

Para el muestreo (no aleatorio) de las participantes se siguieron dos procedimientos diferentes: en el caso de los deportes colectivos, se contactó con los entrenadores de los equipos que respondían a los criterios de la investigación y que aceptaron participar; y en el caso de los deportistas individuales, se contactó con algunas participantes conocidas por el equipo investigador y el resto de la muestra se constituyó por un muestreo de bola de nieve, que consiste en conocer a algunas informantes y conseguir que ellas faciliten el acceso a otras (TAYLOR; BOGDAN, 1986).

La población analizada se corresponde con 46 mujeres residentes en la provincia de Córdoba (España) que respondieron a los tres criterios de inclusión que se plantearon para poder formar parte de la investigación: ser mujer, mayor de 18 años y haber participado en al menos un Campeonato de España (en el caso 
de las deportistas individuales), o que el equipo jugara en una División Nacional o estuviera aspirando al ascenso (en el caso de los deportes colectivos). Los deportes representados en la muestra fueron el fútbol sala, el balonmano, el voleibol, el atletismo y la natación. No se les preguntó directamente cómo se identificaban en términos de orientación sexual porque se les quiso dar flexibilidad para describir su sexualidad según su propio lenguaje (SYKES, 2007).

En cuanto al tipo de caso, siguiendo la clasificación propuesta por Coller (2005), respecto a su alcance, el caso presentado se correspondería con un caso genérico, puesto que ilustra acerca de una característica o fenómeno que se encuentra en otros casos, y con respecto a su naturaleza, con un caso típico. La definición de típico se construye a partir del consenso de opiniones entre las participantes que son plenas conocedoras de la realidad objeto de estudio (STAKE, 2007).

\subsection{INSTRUMENTO}

Para captar los discursos de las deportistas y poder establecer una comparativa según el tipo de deporte practicado, se realizaron cinco grupos focales configurados en función de este criterio. Morgan (1997) señala dos rasgos definitorios de esta técnica: la interacción grupal y la presentación de un tema determinado por el equipo investigador para su discusión. El uso de la situación grupal se utiliza como medio para informarse de la realidad social (GUTIÉRREZ, 2011). Se pretendió crear un espacio en el que las mujeres pudieran expresar libremente sus percepciones y experiencias, ya que según Krueger y Casey (2014) el grupo focal se utiliza para comprender la realidad; no para extraer inferencias, sino para comprender las opiniones particulares que cohabitan en ella. El diseño de los grupos focales intentó facilitar una discursividad abierta y espontánea donde tuvieran cabida todas las contradicciones y diferencias que pudieran existir entre las participantes o entre las distintas posiciones que pudiera adoptar una misma persona (SANZ, 2016).

En cuanto a la composición de los grupos, tres estuvieron formados por jugadoras de deportes de equipo: el primero compuesto por 9 jugadoras de fútbol sala, el segundo por 11 jugadoras de balonmano y el tercero por 10 jugadoras de voleibol. Los otros dos grupos focales estuvieron conformados por las deportistas individuales: uno de mujeres a las que catalogamos como "jóvenes", con edades comprendidas entre los 20 y los 38 años practicantes de atletismo y natación; y otro de mujeres practicantes de atletismo de categoría veterana, con una edad a partir de 45 años. La inclusión de deportistas veteranas nos permite contrastar las percepciones entre mujeres de distintas generaciones y comprobar si tanto el estereotipo de deportista marimacho-lesbiana como la visibilidad de la homosexualidad permanecen ancladas en el imaginario colectivo o bien, han evolucionado a raíz de los cambios sociales acontecidos.

\subsection{PROCEDIMIENTO}

Todos los grupos fueron conducidos por dos investigadoras que ejercieron la función de moderadoras, y estuvieron orientados bajo el mismo guion inicial, que 
se construyó alrededor de los objetivos iniciales de la investigación, pero que se fue enriqueciendo a través de las interacciones de los discursos de las participantes. El trabajo de campo transcurrió entre mayo de 2017 y febrero de 2018. Los dos grupos focales con las deportistas individuales se llevaron a cabo en la Facultad de Ciencias de la Educación de la Universidad de Córdoba, y los de los deportes colectivos se realizaron en las salas de reuniones de los pabellones deportivos donde los equipos entrenaban. La duración de los grupos focales giró en torno a la hora y media. El horario de los grupos focales fue acordado entre las deportistas, el cuerpo técnico de los equipos y el equipo investigador. Todas las participantes se prestaron voluntarias a participar y firmaron un consentimiento informado.

\subsection{ANÁLISIS DE DATOS}

En primer lugar, se procedió a la transcripción de las sesiones, en las que se modificaron los nombres de las participantes para cumplir con los acuerdos de confidencialidad. A continuación, siguiendo la Teoría Fundamentada (STRAUSS; CORBIN; ZIMMERMAN, 2002), se realizó un proceso de codificación abierta en el que se generaron las principales categorías y sus propiedades. De forma simultánea, en la codificación axial las categorías se fueron construyendo y ligando de forma sistemática a las subcategorías hasta que se integraron, y finalmente formaron el esquema explicativo presentado en el apartado de resultados. Durante todo este proceso nos servimos del software NVivo 11 plus.

\section{RESULTADOS Y DISCUSIÓN}

La exposición de los resultados se ha organizado atendiendo a los dos objetivos planteados en esta investigación. Para ilustrar los resultados obtenidos se aportan extractos textuales de los relatos de las mujeres que formaron parte de la muestra.

\subsection{PERCEPCIÓN DE LAS DEPORTISTAS SOBRE CÓMO SON EVALUADAS POR SU ENTORNO SOCIAL EN TÉRMINOS DE FEMINIDAD Y ORIENTACIÓN SEXUAL}

El estereotipo de que las mujeres que practican deporte de competición son lesbianas ha estado siempre presente en los contextos deportivos y de Educación Física (GRIFFIN, 1998; SYKES, 1998; HARRIS, 2005). El deporte siempre se ha contextualizado como un espacio de legitimidad masculina, por lo que las mujeres que se atrevían a adentrarse a niveles competitivos y que presentaban una falta de adherencia de las atletas a las normas tradicionales de feminidad (CAHN, 1993; RIEMER, 1997) eran etiquetadas como lesbianas (KRANE, 1997; KANEMASU; MOLNAR, 2015). Los motivos que pueden conducir a esa percepción de desviación social y, ulteriormente, a ese etiquetado, son diversos: poseer un cuerpo más masculinizado (CAUDWELL, 1999; STIGGER; SILVEIRA, 2010); apartarse de otros cánones estéticos femeninos, como, por ejemplo, llevar el pelo corto y no usar maquillaje (KAUER; KRANE, 2006; FYNES; FISHER, 2016); o llevar a cabo 
desempeños y logros deportivos considerados masculinos por entenderse que trascienden los límites biológicos de la naturaleza femenina (SILVEIRA; VAZ, 2013; 2014). En nuestro estudio encontramos que las deportistas, independientemente de si el deporte estaba socialmente estereotipado o no, se han visto encasilladas dentro del estereotipo de deportista marimacho-lesbiana:

$\mathrm{R}:[\ldots]$, pero he visto que ha habido gente, sobre todo antes, que lo asocia, el hacer mucho deporte y el estar fuerte, a masculino. Entonces se piensan que a lo mejor pues que tus gustos sexuales son otros, que tu orientación sexual es otra, lo asocian a eso... Sí que es verdad que yo creo que ese concepto está cambiando... [...], porque ahora se lleva la mujer no delgada, sino la mujer atlética [Atleta veterana].

B: y antiguamente más..., [...], porque las que hacíamos deporte, éramos todas unos marimachos... [...], si porque se te marque un poco el bíceps ya eres una machorra... [Atleta veterana].

A: hay deportes que desde siempre se han asociado a los hombres, y hay deportes que desde siempre se han asociado a las mujeres, hay deportes femeninos y deportes masculinos... [...], entonces, que por desgracia lo tenemos así en nuestra sociedad. En el momento que, $X$ chicas están en un deporte masculino, se considera que esas chicas van a ser masculinas y... que lo asocian a eso, que no tiene nada que ver... [Jugadora de balonmano]

L: [...], y si juegas al fútbol eres marimacho, si te ponías pantalón, eras marimacho... [Jugadora de fútbol sala].

R: para muchos está asociado el que te vistas con atuendo deportivo y el que dediques muchas horas de tu día, o en tu semana al deporte, está asociado un poco...Lo que pasa es que sí que es verdad que ahora está cambiando... [Atleta veterana]

I: y me han preguntado: ¿pero tú...?, janda!...¿¿Por qué me cuestionas?, ¿Por qué entreno?, pues ya está...[Atleta]

Si se atiende a estos relatos, se desprende que aparte de los motivos que contribuyen a este proceso de etiquetado social citados con anterioridad, se le suma la clasificación tradicional de los deportes en "masculinos" y "femeninos", hecho que defiende ferozmente la tradición heterosexista (HARGREAVES, 1997). Además, y en consonancia con los relatos, los estereotipos culturales asociados a la apariencia (ponerse pantalón y/o atuendo deportivo) y usos del cuerpo (invertir muchas horas entrenando) establecen mecanismos de estigmatización o rechazo social de aquellas imágenes culturales que contradigan el ideal natural heterosexual, en el caso de las mujeres se hace más explícito, el rendimiento deportivo (MOSCOSO; PIEDRA, 2019).

Es evidente que la negatividad implícita en la etiqueta de marimacho-lesbiana minimiza las oportunidades de las mujeres en el deporte y perpetúa sentimientos de hostilidad hacia ellas (KRANE, 1997). Sin embargo, pese a que advierten la existencia de este estereotipo, la mayoría de las mujeres de nuestro estudio expresan no haberse visto condicionadas por este en su práctica deportiva, al igual que en la investigación de Souza, Capraro y Silva (2017). Así, por ejemplo, a través de los relatos de las deportistas observamos cómo el hecho de haberse sentido criticadas por poseer una musculatura que se alejaba de la que se ajusta a la feminidad socialmente exigida (PALZKILL; FISHER, 1990) no ha supuesto una barrera que afectara a la construcción de su propia identidad como mujer y como deportista. Por 
tanto, frente a otros trabajos que han destacado que la amenaza de ser etiquetada como lesbiana y el estigma resultante ha llevado a muchas atletas a disociarse de las prácticas asociadas a las lesbianas (BLINDE; TAUB, 1992) tales como cambiarse a otro deporte considerado socialmente como más femenino como el voleibol (KAUER; KRANE, 2006), o modificar tanto su forma de vestir como el uso de maquillaje para acercarse a la feminidad hegemónica (HARGREAVES, 1997; FYNES; FISHER, 2016), las integrantes de nuestra muestra mantienen, por lo general, un discurso de resistencia a este respecto:

\begin{abstract}
M: sí marimachos, exactamente, ahí va la marimacho... A mí siempre me ha dado igual. Aparte, es que yo soy una tía sin complejos, que me da igual lo que dijera la gente; yo seguía compitiendo... [Atleta veterana].

D: [...] Entonces, a lo mejor a ti te las ve [las piernas] tu abuela, por ejemplo: "qué piernas de hombre". O muchas veces te decían, incluso los niños, que son muy cabrones, te decían: "eres un marimacho", "tienes cuerpo de hombre". Y entonces yo veía esas piernas que empezaban a correr [...], y yo decía: "pues yo quiero tener mis piernas fuertes, me encuentro muy bien con mi cuerpo así". [Jugadora de balonmano].
\end{abstract}

Este resultado se encuentra en consonancia con los obtenidos por Howe (2001) en una muestra de jugadoras de rugby que, igualmente, mantenían un discurso de resistencia frente a este estereotipo. Así pues, en nuestro caso de estudio, y en línea también con lo encontrado en algunos trabajos previos (CAHN, 1993), el deporte se configura como un espacio que recoloca a las mujeres con identidades sexuales diversas, en un nexo alternativo de significados de género, permitiéndoles ser ellas mismas, expresar su género o su sexualidad de una manera no convencional y redefinir la feminidad en sus propios términos (ALTMANN; REIS, 2013).

No obstante, en paralelo a este relato como víctimas (resistentes) de estos procesos de etiquetado, las participantes en los grupos perciben que en los últimos tiempos viene produciéndose un cambio cultural a este respecto. Un cambio que, a su parecer, está conduciendo a una mayor aceptabilidad social hacia la imagen de la mujer deportista, de manera que, poco a poco, esta empieza a desvincularse de la feminidad hegemónica:

A: [...] ahora es una moda, [...], yo que sé, está más socialmente aceptado, por ejemplo, la persona que hace deporte. Hay mucha más gente ahora que hace deporte que la que no lo hace, entonces, pues las mujeres, igualmente, pues se ve que está más aceptada. Es distinto. [Jugadora de balonmano].

R: [...] las mujeres en general han perdido el miedo..., porque yo trabajo en un gimnasio y escucho este tipo de conversación [...] A las mujeres antes les daba mucho miedo el hecho de coger una pesa, entrenar, ponerse fuerte, marcar los músculos, y ahora tienen como motivación..., les empieza a gustar eso, y ahora las mujeres van mucho a..., "me voy a poner fuerte"... [Jugadora de balonmano].

Resulta relevante el hecho de que las participantes, independientemente del tipo de deporte practicado (colectivo-individual, estereotipado o no) y de la edad, mantienen un discurso homogéneo respecto a las cuestiones tratadas en este apartado. Este hecho coincide con los resultados encontrados en el ámbito que se explora a continuación, referido a la visibilidad de la homosexualidad en el entorno social de las mujeres deportistas. 


\subsection{SITUACIÓN Y MANEJO DE LA VISIBILIDAD DE LA HOMOSEXUALIDAD EN EL ENTORNO SOCIAL DE LA MUJER DEPORTISTA}

\subsubsection{Percepciones internas de las deportistas}

Se exponen bajo este epígrafe las percepciones que tienen las deportistas analizadas sobre la situación de visibilidad de la homosexualidad tanto en el espacio deportivo en el que desarrollan su entrenamiento, como fuera de él. Según Foucault (2006), la sexualidad está profundamente asociada a la pareja (heterosexual), legítima y procreadora, lo que provoca que muchas mujeres se vean obligadas a encubrir sus relaciones homosexuales y se dificulta, en consecuencia, una vivencia despreocupada de la sexualidad. Griffin (1992b) identificó a deportistas pertenecientes al colectivo LGTB que se veían sometidas a tratos homofóbicos por parte de sus compañeros de equipo, convirtiéndose el ámbito deportivo en un espacio en el que se sentían incómodas por ser como eran. En contraste, en nuestro estudio encontramos, en primer lugar, que las deportistas analizadas reconocen, aceptan y visibilizan su sexualidad en el entorno deportivo:

C: [...] llega un momento en el que "a mí me gusta esto, o me aceptáis o me da igual", ¿sabes?, [...], entonces, pues...yo lo tengo con toda naturalidad, ¿sabes?, que yo lo digo y me da igual, pero a lo mejor hay una persona que le molesta o que no quiere que se sepa... [Jugadora de fútbol sala].

Este discurso refuerza los hallazgos de Krane (1997), que encontró que las chicas que habían sabido lidiar con las reacciones homonegativas de su entorno adoptaron una identidad lesbiana positiva. La deportista en esta fase percibe su identidad lesbiana como un componente esencial de su propia identidad.

En segundo lugar, hallamos que todas las deportistas analizadas incluso percibían el entorno deportivo, independientemente de si el deporte estaba estereotipado o no, como un dispositivo de normalización de la homosexualidad. En línea con algunos trabajos previos (KAUER; KRANE, 2006; RAVEL; RAIL, 2006), la homosexualidad es percibida como una cuestión normalizada entre las deportistas y no supone ningún tabú entre ellas. Es más, el contexto deportivo se les presenta como un espacio de respeto en el que pueden ser ellas mismas, hablar de sus relaciones homosexuales sin miedo a sentirse rechazadas, transgredir la noción hegemónica de feminidad y las convenciones sociales relativas a la heteronorma:

P: yo creo que, a día de hoy, eso [la homosexualidad normalizada en el contexto deportivo] sigue siendo así en ese sentido..., el hecho de estar metida en este ambiente es lo más normal. [Jugadora de futbol sala].

M: pues yo coincidí con una [compañera homosexual] en el equipo de Superliga y ella tenía su pareja y era totalmente abierta... [Jugadora de voleibol]

C: la que nosotros hemos tenido [su compañera homosexual] lo hace, totalmente. La que nosotros hemos tenido en nuestro grupo, se expresa totalmente... [Atleta].

L: no, quiero decir que ella [su compañera homosexual] lo ha dado como normal, lo ha normalizado y la gente lo ha tomado normalizado en este periodo. [Atleta].

A: yo que sé..., yo he estado en varios vestuarios y he tenido compañeras que han tenido parejas dentro de ese equipo y yo lo he visto normal... [Jugadora de balonmano]. 
En consonancia con lo anterior, quisimos indagar en un hecho que, según Gough (2007) y Bury (2012), suele ocurrir cuando las deportistas homosexuales deciden hacer pública su sexualidad: los conflictos que se pueden generar dentro del equipo o grupo de entrenamiento. Así, por ejemplo, Krane (1997) identificó a deportistas que se habían sentido rechazadas cuando sus compañeras se enteraron de que eran lesbianas porque pensaban que las observaban en el vestuario mientras se cambiaban, Harris (2005) encontró que las deportistas homosexuales eran objeto de bromas cuando el resto del equipo conoció su orientación sexual, y Anderson y Bullingham (2013) hallaron a chicas que se negaron a compartir habitación con compañeras de equipo cuando se enteraron de que eran homosexuales. Pero en nuestra investigación, y en línea con estudios más recientes, constatamos claras diferencias con respecto a los trabajos citados, ya que incluso en el caso del voleibol, un deporte estereotipado como femenino en el contexto de la investigación y en el que, en principio, la presencia de chicas homosexuales debería estar más estigmatizada, la aceptación de la homosexualidad por parte de las compañeras es clara y no condiciona de manera negativa la convivencia entre las compañeras: "M: yo las que he tenido [compañeras homosexuales en su mismo equipo]...normal..., [...], yo hasta me quedaba a dormir, imagínate... Muy buena, vamos... [Jugadora de voleibol]."

Como señalábamos, estos datos son consistentes con estudios que, en los últimos años, atenúan la presencia de actitudes homonegativas en el deporte hacia los deportistas homosexuales. Es el caso del trabajo de Fynes y Fisher (2016), en el que, a pesar de identificar a algunas deportistas homosexuales que ocultaron su identidad lesbiana, la mayoría manifestó que sus compañeras las acogían y respetaban cuando ellas decidían hacer pública su sexualidad.

La información producida en nuestra investigación indica que existe un clima de aceptación y respeto por la sexualidad de las jugadoras en el seno del ámbito deportivo. Los relatos de las dos deportistas extranjeras refuerzan este clima de normalización en el contexto español frente al que han percibido en sus países nativos. Una jugadora procedente de Rumanía enfatiza:

\begin{abstract}
R: luego al final te abres de mente porque yo, por ejemplo, mi caso, cuando yo llegué a España y empecé a ver esas cosas, era como: "¡oh Dios mío, qué vergüenza...!", porque en mi país era igual que ella [refiriéndose al discurso de su compañera de equipo de origen chileno]:"eres lesbiana y venga, muérete”, en plan así igual, entonces cuando yo llegué aquí era como:"¿esto qué es?", [...] pero claro eso luego lo vas aprendiendo con la gente que vas conociendo y te abres y dices: "espérate, que esto no es lo que te han dicho..., es lo que tú exploras, lo que tú ves" [Jugadora de balonmano].
\end{abstract}

Por la intervención de esta jugadora se puede observar cómo el rechazo que sentía inicialmente, debido a los estereotipos que había aprendido durante su infancia y adolescencia sobre las mujeres lesbianas, se acaba suavizando y la jugadora empieza a crear su propio sistema de creencias, tal y como identificó Riemer (1997). En concordancia con el relato de esta jugadora encontramos los estudios de Mennesson y Clément (2003) y de Kauer y Krane (2006), en los que identificaron a jugadoras que en un principio presentaban una tendencia homonegativa, pero que cuando empezaron a convivir en un espacio en el que homosexualidad era aceptada 
y establecieron lazos afectivos de amistad, aprendieron a romper la creencia de que la homosexualidad era una enfermedad o una maldición. El deporte, en ese sentido, es percibido como un vehículo para la acción social transgresiva y como un espacio seguro en el cual las atletas homosexuales ayudaban a sus compañeras heterosexuales a romper con la heteronormatividad y a aceptar la sexualidad de sus compañeras (FINK et al. 2012; MELTON; CUNNINGHAM, 2012).

Sin embargo, el relato de otra jugadora de balonmano extranjera (chilena en este caso) nos revela también que, en otros ámbitos de la vida como el escolar, el laboral, el familiar o el tiempo de ocio, las jugadoras homosexuales prefieren optar por estrategias de invisibilidad. Por ejemplo, Riemer (1997) identificó que las jugadoras de softball descubren su verdadera sexualidad en el contexto deportivo pero que en otros ámbitos vitales se lo pueden contar a una persona concreta pero no lo visibilizan con naturalidad. En consonancia con lo anterior, Ravel y Rail (2006) encontraron que las deportistas salían del armario en algunas áreas de su vida como la deportiva, pero que en otras permanecían escondidas. Al hilo de lo anterior, Kanemasu y Molnar (2015) encontraron que las jugadoras de rugby homosexuales encuentran en el espacio deportivo un refugio seguro para mostrar su verdadera sexualidad, sin embargo, en otras facetas de su vida la mantienen escondida por miedo a la represión social.

C: yo entré, por ejemplo, con la duda..., yo dije, mira, llegué a España..., en el equipo..., buah, [50-50], súper normal, no sé qué...y cuando entré en la Universidad, dije: “¿será igual?". Me hice la pregunta: “¿será igual?" Porque..., y no, no era igual, en la clase se oculta, pero en el vestuario no. [Jugadora de balonmano].

Las percepciones de ambas jugadoras, de una procedencia cultural muy dispar, inducen a pensar que la situación para las mujeres homosexuales en sus países de origen es más desalentadora en términos de aceptabilidad social de la que han percibido en España, al tiempo que subrayan los avances logrados en este ámbito en este país. Aun así, pese a estos avances, en España persisten actitudes negativas hacia la homosexualidad debido a que la heterosexualidad está institucionalizada y convierte en antinaturales o tabúes otras formas de sexualidad (PIEDRA, 2015). Así es percibido por la totalidad de las participantes en nuestro estudio:

\footnotetext{
E: pero es que España era así también hace no tanto... [Jugadora de balonmano]

L: a lo mejor todavía no está..., aunque hayamos dado un paso y ya vemos muchas parejas tanto de hombres como de mujeres... [Atleta veterana].

F: a nivel de chicas sí, pero sigue siendo un tabú y eso vamos, va a ser siempre un tabú..., [...], tú no lo ves..., pero la mayoría sabes que sí. [Jugadora de balonmano].
}

Estos datos van en la línea con los encontrados por Kauer y Krane (2006), en los que a pesar de que las deportistas se atrevían a transgredir el mandato de la heterosexualidad y perciben que el cambio social estaba teniendo lugar dentro de los equipos deportivos, era difícil predecir si uno más amplio estaba sucediendo dentro del deporte femenino. Muchas deportistas optan por prácticas de silencio y mantienen su identidad oculta en el armario (SYKES, 1998) con el fin de mantener 
la heteronorma o se suman, a lo que Anderson y Bullingham (2013) presentan como el don't ask, don't tell, que representa la actitud de que todo el entorno conoce la sexualidad predominante de ese espacio deportivo concreto, pero nadie lo reconoce de forma pública.

\subsubsection{Papel de la familia}

Con respecto al papel de la familia, las participantes afirmaron haberse sentido apoyadas por el núcleo familiar a lo largo de toda su carrera deportiva, reconocen la suerte que han tenido y la importancia del soporte emocional y afectivo familiar cuando se practica un deporte estereotipado masculino de alto rendimiento: "N: [...] es muy importante eso, la familia, que te apoye. [Jugadora de fútbol sala]."

En contraposición, encontramos que existe una percepción general de las jugadoras de fútbol sala analizadas acerca de la opinión de un gran número de familias de su entorno cercano, cuando sus hijas eligen una práctica deportiva estereotipada como masculina, aspecto que ya señaló Griffin (1998) y que se concreta en la creencia de que ciertos deportes vuelven o convierten en lesbianas a las chicas:

$P:[\ldots]$ mi padre lleva un club femenino de categorías base de niñas muy pequeñitas y ha tenido muchos problemas por parte de muchos padres $y$ de muchas familias, de no querer apuntar a sus niñas [...] porque en ese equipo hay gente homosexual, y quizás el temor de los padres es [...], por miedo a que mi niña..., no es quizás que se relacione, sino por miedo a que mi niña se convierta en homosexual. [Jugadora de fútbol sala].

En este relato se puede observar el miedo de las familias a que las niñas no interioricen la sexualidad hegemónica y consideran al deporte categorizado como masculino un escenario en el que sus hijas pueden descubrir otras formas de sexualidad y sentirse atraídas por sus propias compañeras de equipo.

En investigaciones más recientes, Kanemasu y Molnar (2015) encontraron a jugadoras de rugby a las que sus familias las habían agredido y expulsado del domicilio familiar por participar en una actividad deportiva que, a su parecer, contradecía las normas de género.

\section{CONCLUSIONES}

Una vez expuestos y analizados los resultados del estudio, nos unimos a la afirmación de Piedra (2015) y constatamos que, a pesar de los avances sociales producidos, los estudios acometidos en países occidentales, España incluida, muestran un panorama diverso que oscila desde una cierta normalización a la permanencia de un clima hostil hacia la diversidad sexual.

En primer lugar, a través de los relatos de las deportistas estudiadas, observamos que el estereotipo de marimacho y su consiguiente asociación al lesbianismo (RIEMER, 1997; CAUDWELL, 1999; KAUER; KRANE, 2006) continúa vigente en el imaginario colectivo, ya que todas, en algún momento de su vida, se han sentido etiquetadas como tal. Sin embargo, relatan haber llevado a cabo una actitud y un discurso de resistencia en este sentido. Afortunadamente, perciben una 
mayor aceptabilidad social del cuerpo de la mujer deportista que no responde a la tradicional feminidad hegemónica.

Por otra parte, la nueva visibilidad y aceptación social de las relaciones homosexuales derivadas, entre otras cuestiones, de las leyes que se están aprobando en los países occidentales (FREIXAS; LUQUE; REINA, 2010) se configura como un punto de partida de carácter institucional que necesitan las personas para conseguir la consiguiente normalización y aceptación en el contexto deportivo. El deporte se configura como un escenario en el que las deportistas homosexuales pueden descubrir y reconocer su sexualidad al percibirlo como un refugio seguro (STOELTING, 2011) en el que pueden explorar su identidad de género y lo que significa ser mujer y deportista (RATTS et al. 2013).

La cultura deportiva representa un microcosmos de la sociedad que refleja los cambios tanto en las políticas de gobierno como en la opinión pública (HALBROOK; WATSON; VOELKER, 2018). A pesar de que la sociedad española ha evolucionado en estos últimos años hacia un incremento en los niveles de aceptación hacia las personas pertenecientes al colectivo LGTBI, aún persisten formas de rechazo cognitivo y afectivo (VÉLEZ; PIEDRA, 2018). Este hecho coloca a España en un nivel de pseudo-inclusividad que se identifica como una salida políticamente correcta desde el rechazo a la diversidad sexual (con la implicación negativa de ser homófobo) junto con la negativa a aceptar a las minorías sexuales (PIEDRA; GARCÍA-PÉREZ; CHANNON, 2017). Esta situación pseudo-inclusiva de la homosexualidad es percibida y catalogada por las deportistas del estudio como tabú, al ser aceptada y conocida en el espacio privado deportivo, pero invisibilizada en el espacio público.

La familia juega un papel clave en la estructuración de la identidad de la deportista, ofreciéndole el apoyo afectivo y emocional necesario para la práctica deportiva de alto rendimiento. Nuestros resultados parecen indicar que, a pesar de la evidencia de que el homonegativismo está disminuyendo (ANDERSON; BULLINGHAM, 2013), la homosexualidad sigue estigmatizada (PETTY; TRUSSELL, 2017) y su relación con el deporte estereotipado masculino cuando es practicado por mujeres, es percibido por los entornos familiares cercanos a nuestra muestra, como un factor que pone en riesgo que sus hijas se alejen de la sexualidad considerada como socialmente aceptada.

Los resultados encontrados invitan a un examen más profundo que refuerce en un sentido o en otro los hallazgos obtenidos. El empleo, por una parte, de una metodología que permita cuantificar algunas de las percepciones que emergen en los discursos analizados y, por otra, de una metodología cualitativa pero centrada en los agentes sociales (familia, entrenadores, parejas, etc.), pueden ofrecer un conocimiento más amplio y ajustado de una realidad que acontece diariamente en el contexto deportivo. 


\section{REFERENCIAS}

ALTMANN, Helena; REIS, Heloisa Helena Baldy. Futsal feminino na América do Sul: trajetórias de enfrentamentos e de conquistas. Movimento, v. 19, n.3, p. 211-232, 2013.

ANDERSON, Eric; BULLINGHAM, Rachael. Openly lesbian team sport athletes in an era of decreasing homohysteria. International Review for the Sociology of Sport, v.50, n. 6, p. 647-660, 2013.

BLINDE, Elaine M.; TAUB, Diane E. Women athletes as falsely accused deviants: Managing the lesbian stigma. Sociological Quarterly, v. 33, n.4, p. 521-533, 1992.

BURY, Jonah. Can a footballer be gay? The problem of (homo)sexuality and coming out in English professional football. In: ADAMS, Andrew; SPRACKLAN, Karl (ed.). Sport and leisure ethics, rights and social relationships. Eastbourne: Leisure Studies Association, 2012. p. 79-95.

CAHN, Susan K. From the" muscle moll" to the" butch" ballplayer: Mannishness, lesbianism, and homophobia in US women's sport. Feminist studies, v. 19, n. 2, p. 343-368, 1993.

CAUDWELL, Jayne. Women's football in the United Kingdom: Theorizing gender and unpacking the butch lesbian image. Journal of Sport and Social Issues, v. 23, n. 4, p. 390402, 1999.

COLLER, Xavier. Estudio de casos. Madrid: Centro de Investigaciones Sociológicas, 2005.

DAVIS-DELANO, Laurel R. Sport as context for the development of women's same-sex relationships. Journal of Sport and Social Issues, v. 38, n. 3, p. 263-285, 2014.

DOWLING, Fiona. Teacher educators' gendered workplace tales. In: PFISTER, Gertrud; SISJORD, Mari Kristin (ed.). Gender and Sport. Changes and Challenges. Münster: Waxmann, 2013. p. 217-231.

FASTING, Kari; SCRANTON, Sheila. The myth of masculinization of the female athlete: The experiences of European sporting women. In: CONGRESS OF THE NORTH AMERICAN SOCIETY FOR THE SOCIOLOGY OF SPORT. Toronto, 1997.

FINK, Janet et al. Playing it out. Journal for the Study of Sports and Athletes in Education, v. 6, n. 1, p. 83-106, 2012.

FOUCAULT, Michael. Historia de la sexualida: La voluntad de saber. Madrid: Siglo XXI, 2006.

FREIXAS, Anna; LUQUE, Bárbara; REINA, Amalia. Secretos y silencios en torno a la sexualidad de las mujeres mayores. Debate Feminista, v. 42, p. 35-51, 2010.

FYNES, Jamie M.; FISHER, Leslee. A. Is authenticity and integrity possible for sexual minority athletes? Lesbian student-athlete experiences of US NCAA Division I sport. Women in Sport and Physical Activity Journal, v. 24, n. 1, p. 60-69, 2016.

GOUGH, Brendan. Coming out in the heterosexist world of sport: A qualitative analysis of web postings by gay athletes. Journal of gay \& lesbian psychotherapy, v.11, n.1-2, p.153$174,2007$.

GRIFFIN, Pat. Changing the game: Homophobia, sexism, and lesbians in sport. Quest, v. 44, n. 2, p. 251-265, 1992. Disponible en: http://doi:10.1080/00336297.1992.10484053. Acceso en: 24 ene.2019. 
GRIFFIN, Pat. Strong women, deep closets: Lesbians and homophobia in sport. Bethesda: Human Kinetics, 1998.

GROV, Christian et al. Race, ethnicity, gender, and generational factors associated with the coming-out process among gay, lesbian, and bisexual individuals. Journal of sex research, v.43, n.2, p.115-121, 2006. Disponible en: http://doi:10.1080/00224490609552306. Acceso en: 14 ene.2019.

GUTIÉRREZ, Jesús. Grupo de discusión:¿ prolongación, variación o ruptura con el focus group?. Cinta de moebio, n.41, p.105-122, 2011.

HALBROOK, Meghan K.; WATSON, Jack C.; VOELKER, Dana K. High School Coaches' Experiences with Openly Lesbian, Gay, and Bisexual Athletes. Journal of homosexuality, v.66, n.6, p.838-856, 2018. Disponible en: http://doi:10.1080/00918369.2017.1423222. Acceso en: 17 ene.2019.

HARGREAVES, Jennifer. Women's boxing and related activities: Introducing images and meanings. Body \& Society, v.3, n.4, p.33-49, 1997. Disponible en: http://doi:10.1177/13570 34x97003004002. Acceso en: 18 ene. 2019.

HARRIS, John. The image problem in women's football. Journal of Sport and Social Issues, v.29, n.2, p.184-197, 2005. Disponible en: http://doi:10.1177/0193723504273120. Acceso en: 3 feb. 2019.

HOWE, P. David. Women's rugby and the nexus between embodiment, professionalism and sexuality: An ethnographic account. Football Studies, v.4, n. 2, p. 77-92, 2001.

JAEGER, Angelita Alice et al. Formação profissional em educação física: homofobia, heterossexismo e as possibilidades de mudanças na percepção dos (as) estudantes. Movimento, v.25, 2019. Disponible en: https://doi.org/10.22456/1982-8918.88681. Acceso en: 16 ene. 2019 .

JUSTER, Robert-Paul, et al. Retrospective coping strategies during sexual identity formation and current biopsychosocial stress. Anxiety, stress, \& coping, v.29, n.2, p. 119-138, 2016. Disponible en: htts://doi.org/10.1080/10615806.2015.1004324. Acceso en: 23 ene.2019.

KANEMASU, Yoko; MOLNAR, Gyozo. Double-trouble: Negotiating gender and sexuality in post-colonial women's rugby in Fiji. International Review for the Sociology of Sport, v.52, n.4, p.430-446, 2015. Disponible en: http://doi:10.1177/1012690215602680. Acceso en: 27 ene. 2019.

KAUER, Kerrie J.; KRANE, Vikki. "Scary dykes" and" feminine queens": Stereotypes and female collegiate athletes. Women in Sport \& Physical Activity Journal, v.15, n.1, p.42-58, 2006. Disponible en: http://doi:10.1123/wspaj.15.1.42. Acceso en: 30 ene. 2019.

KIVEL, Beth D.; KLEIBER, Douglas A. Leisure in the identity formation of lesbian/gay youth: Personal, but not social. Leisure Sciences, v.22, n.4, p. 215-232, 2000.

KRANE, Vikki. Homonegativism experienced by lesbian collegiate athletes. Women in Sport and Physical Activity Journal, v.6, n.2, p.141-163, 1997.

KRUEGER, Richard A.; CASEY, Mary Anne. Focus groups: A practical guide for applied research. Thousand Oaks: Sage, 2014.

LENSKYI, Helen. Sexuality and femininity in sport contexts: Issues and alternatives. Journal of Sport and Social Issues, v.18, n.4, p.356-376, 1994. Disponible en: http:// doi:10.1177/019372394018004005. Acceso en: 4 ene. 2019. 
MARTÍN, Montse.; SOLER, Susanna.; VILANOVA, Anna. Género y deporte. In: GARCÍA FERRANDO, Manuel; PUIG, Nuria; LAGARDERA, Francisco.; LLOPIS, Ramón; VILANOVA, Anna. (ed.). Sociología del deporte. Madrid: Alianza Editorial, 2017. p. 97-123.

MELTON, Nicole; CUNNINGHAM, George. When identities collide. Journal for the Study of Sports and Athletes in Education, v.6, n.1, p.45-66, 2012. Disponible en: http:// doi:10.1179/ssa.2012.6.1.45. Acceso en: 8 feb. 2019.

MENNESSON, Christine; CLÉMENT, Jean-Paul. Homosociability and homosexuality: The case of soccer played by women. International Review for the Sociology of Sport, v.38, n.3, p. 311-330, 2003.

MORGAN, David L. Focus groups as qualitative research. Thousand Oaks. Cal: Sage, 1997.

MOSCOSO, David; PIEDRA, Joaquín. El colectivo LGTBI en el deporte como objeto de investigación sociológica. Estado de la cuestión. Revista Española de Sociología, v. 28, n.3, 2019. Doi: http://dx.doi.org/10.22325/fes/res.2019.06. Acceso en: 10 feb.2020.

OXFORD, Sarah; MCLACHLAN, Fiona. "You Have to Play Like a Man, But Still be a Woman": Young Female Colombians Negotiating Gender Through Participation in a Sport for Development and Peace (SDP) Organization. Sociology of Sport Journal, v.35, n.3, p. 258-267, 2018. Disponible en: http://doi:10.1123/ssj.2017-0088. Acceso en: 7 feb. 2019.

PALZKILL, Birgit; FISHER, Angela. Between gymshoes and high-heels-The development of a lesbian identity and existence in top class sport. International review for the sociology of sport, v.25, n.3, p. 221-234, 1990. Disponible en: http://doi:10.1177/101269029002500304. Acceso en: 11 feb. 2019.

PETTY, Lisa; TRUSSELL, Dawn. Experiences of identity development and sexual stigma for lesbian, gay, and bisexual young people in sport: 'Just survive until you can be who you are'. Qualitative Research in Sport, Exercise and Health, v.10, p. 1-14, 2017. Disponible en: http://doi.1080/2159676X.2017.1393003. Acceso en: 13 feb.2019.

PIEDRA, Joaquín. Gays y lesbianas en el deporte: discurso de jóvenes universitarios españoles en torno a su aceptación. Movimento, 2015, v. 21, n. 4, p. 1067-1081, 2015.

PIEDRA, Joaquin; GARCIA-PEREZ, Rafael; CHANNON, Alexander G. Between homohysteria and inclusivity: Tolerance towards sexual diversity in sport. Sexuality \& Culture, v. 21, n.4, p. 1018-1039, 2017. Disponible en: http://doi:10.1007/s12119-017-9434-x. Acceso en: 14 feb. 2019.

RATTS, Manivong J. et al. Safe space programs in K-12 schools: Creating a visible presence of LGBTQ allies. Journal of LGBT Issues in Counseling, v.7, n.4, p. 387-404, 2013.

Disponible en: http://doi:10.1080/15538605.2013.839344. Acceso en: 22 ene. 2019.

RAVEL, Barbara; RAIL, Geneviève. The Lightness Of BeingGaie' Discursive Constructions of Gender and Sexuality in Quebec Women's Sport. International Review for the Sociology of Sport, v.41, n.3-4, p. 395-412, 2006. Disponible en: http:// doi:10.1177/1012690207080239. Acceso en: 28 ene. 2019.

REMAFEDI, Gary. Homosexual youth. Journal of the American Medical Association, v. 258, n. 2, p. 222-225, 1987.

RIEMER, Brenda A. Lesbian identity formation and the sport environment. Women in Sport and Physical Activity Journal, v.6, n.2, p. 83-107, 1997. 
ROBINSON, Joseph P.; ESPELAGE, Dorothy L.; RIVERS, lan. Developmental trends in peer victimization and emotional distress in LGB and heterosexual youth. Pediatrics, v. 131, n.3, p. 423-430, 2013.

SANZ, Cristina. Identidades masculinas: estereotipos de género y participación en la educación física, la actividad física y el deporte. (Tesis) -Facultad de Educación, Universidad Complutense, 2016.

SILVEIRA, Viviane; VAZ, Alexandre. Doping e controle de feminilidade no esporte. Cadernos Pagu, n. 42, p. 447-475, 2013. Disponible en: http://dx.doi.org/10.1590/01048333201400420447. Acceso en: 14 feb.2020.

SILVEIRA, Viviane; VAZ, Alexandre. Corpo feminino no esporte: entre heterossexualidade compulsória e lesbofobia. Revista Brasileira de Ciências do Esporte, v. 36, n.2, p. 212222, 2014. Disponible en: http://www.rbce.cbce.org.br/index.php/RBCE/article/view/2128. Acceso en: 15 feb. 2020.

SOUZA, Maria Thereza; CAPRARO, André Mendes; SILVA, Marcelo Moraes e. Habilidosas e bonitas: as considerações de duas atletas de futebol sobre a formação de suas identidades. Movimento, v.23, n.3, p.883-894, 2017.

STAKE, Robert. Investigación con estudio de casos. Madrid: Ediciones Morata, 2007.

STOELTING, Suzanne. Disclosure as an interaction: Why lesbian athletes disclose their sexual identities in intercollegiate sport. Journal of Homosexuality, v.58, n.9, p.1187-1210, 2011.

STIGGER, Marco Paulo; SILVEIRA, Raquel da. Ocio y homosexualidad: un estudio etnográfico sobre el asociativismo deportivo de mujeres, en el contexto de un deporte dicho masculino. Polis - Revista Latinoamericana, v.9, n.26, p. 133-155, 2010. http://dx.doi. org/10.4067/S0718-65682010000200007.

STRAUSS, Anselm L.; CORBIN, Juliet; ZIMMERMAN, Eva. Bases de la investigación cualitativa: técnicas y procedimientos para desarrollar la teoría fundamentada. Antioquía: Editorial Universidad de Antioquía, 2002.

SYKES, Heather. Turning the closets inside/out: Towards a queer-feminist theory in women's physical education. Sociology of Sport Journal, v.15, n.2, p.154-173, 1998.

SYKES, Heather. Queering theories of sexuality in sport studies. In: CAUDWELL, Jane (ed.). Sport, sexualities and queer/theory. Londres: Routledge, 2007. p. 25-44.

TAYLOR, Steve; BOGDAN, Robert. Introducción a los métodos cualitativos de investigación:- La búsqueda de significados. Buenos Aires: Paidos, 1986.

VELEZ, Luisa; PIEDRA, Joaquin. Does sexuality play in the stadium? Climate of tolerance/ rejection towards sexual diversity among soccer players in Spain. Soccer \& Society, p. 1-10, 2018. Disponible en: http://doi:10.1080/14660970.2018.1446002. Acceso en: 15 ene. 2019. 